\title{
ÉDITORIAL
}

\section{L'irradiation médicale, le vrai, le faux}

Dans sa lettre à la rédaction, Jean-Claude Nénot signale que la CIPR, à l'origine focalisée sur l'irradiation médicale, a principalement axé ses efforts sur le secteur nucléaire dès sa réorganisation en 1950 . Aujourd'hui le Comité 3 , chargé de la médecine, où l'on trouve l'ancien président de la SFRP, le professeur Jean-Marc Cosset, a été complètement redynamisé depuis quatre ans par son nouveau président, le professeur Fred Mettler. Les publications s'enchaînent, avec un caractère concret: lors de sa réunion annuelle, en septembre 2001, on nous a annoncé des guides pratiques, des posters, bref un réel effort de communication auprès des professionnels de la santé. Il était temps.

La deuxième lettre à la rédaction montre l'utilité de cet effort. Les mammographies sont des actes médicaux que certains, sans bien en connaître les différents aspects, critiquent, avec comme seul résultat l'affolement de femmes qui ne savent plus que faire. C'est donc avec une grande satisfaction que notre revue accueille cette lettre, écrite par de vrais spécialistes. Toute activité humaine présente un risque, et si il est vrai qu'il ne faut pas cesser l'effort de réduction des doses, le risque induit par une mammographie correctement prescrite reste faible. Il faut rassurer les intéressées et faire stopper ces rumeurs qui ne peuvent que nuire au plus grand nombre.

Une fois de plus notre rubrique « lettre à la rédaction» rempli bien son rôle, j'espère que notre communauté, bien vivante, trouvera d'autres thèmes d'échanges pour d'autres lettres tout aussi vivantes.

Je vous conseille aussi de lire la revue des livres, nos amis de la section «Rayonnement non-ionisants » y sont très justement à l'honneur.

À très bientôt

Henri Métivier

Président du comité de la revue 